\title{
New Trends in Disentangling the Spectra of Multiple Stars
}

\author{
Petr Hadrava \\ Astronomický ústav, Akademie věd České republiky, CZ-251 65 Ondřejov, Czech Republic \\ email: had@sunstel.asu.cas.cz
}

\begin{abstract}
The method of spectra disentangling has been applied in many studies on different stellar systems up to the distance of Andromeda Galaxy. In some of these applications the underlying assumptions are not precisely satisfied. This is why new generalizations of the method are needed. Ways to overcome these problems are discussed here.
\end{abstract}

Keywords. techniques: spectroscopic, (stars:) binaries: spectroscopic

\section{Introduction}

The mutual interaction of components of binary and multiple stellar systems yields an admirable chance to determine the basic parameters of stars, the knowledge of which is crucial for understanding many problems in astrophysics. At the same time, the interaction complicates the physics of the component stars as well as the processing of observational data. Tricky models and methods are thus needed to get the desirable information from the observations of different kind.

A basic example of such procedures and their difficulties can be met in spectroscopy of binaries: the solution of radial-velocity curves (with an additional information from photometry) gives the dimensions of the systems and the masses of components, but it requires to distinguish the spectra of component stars. On the other hand, the components' spectra, the correspondence of which with the masses and other parameters of stars is of a particular interest, can be decomposed from their observed superpositions, if the radial velocities (RVs hereafter) are known. The problems are thus mutually entangled.

\section{Disentangling $=$ decomposition of spectra + fitting of parameters}

Various methods enabling to perform one or the other of these tasks have been developed. If the spectral lines of binary components are blended, methods like crosscorrelation (cf., Hill 1993), two-dimensional cross-correlation (Zucker \& Mazeh 1994) or method of broadening function (Rucinski 1992) are needed to measure RVs of component stars. These methods are based on the use of template spectra, which should correspond to the spectra of components of the binary system (cf., Figure 1). The orbital parameters can be then obtained by RV-solution using a straightforward fit, but it is preferable to solve them simultaneously with light-curves or other types of data (cf., Wilson 1979; Kallrath \& Milone 1999; Hadrava 2004b, 2005).

To get spectra of binary components for a detailed analysis, several methods of decomposition have been developed, which use the relative Doppler shifts for known RVs. The most successful one is the method of tomographic separation (Bagnuolo \& Gies 1991).

Intuitively, it is obvious, that - at least by some iterative procedure - the information on both the RVs and component spectra can be extracted from the observed spectra, in which it is entangled. This is the principle of methods of disentangling (Simon \& Sturm 


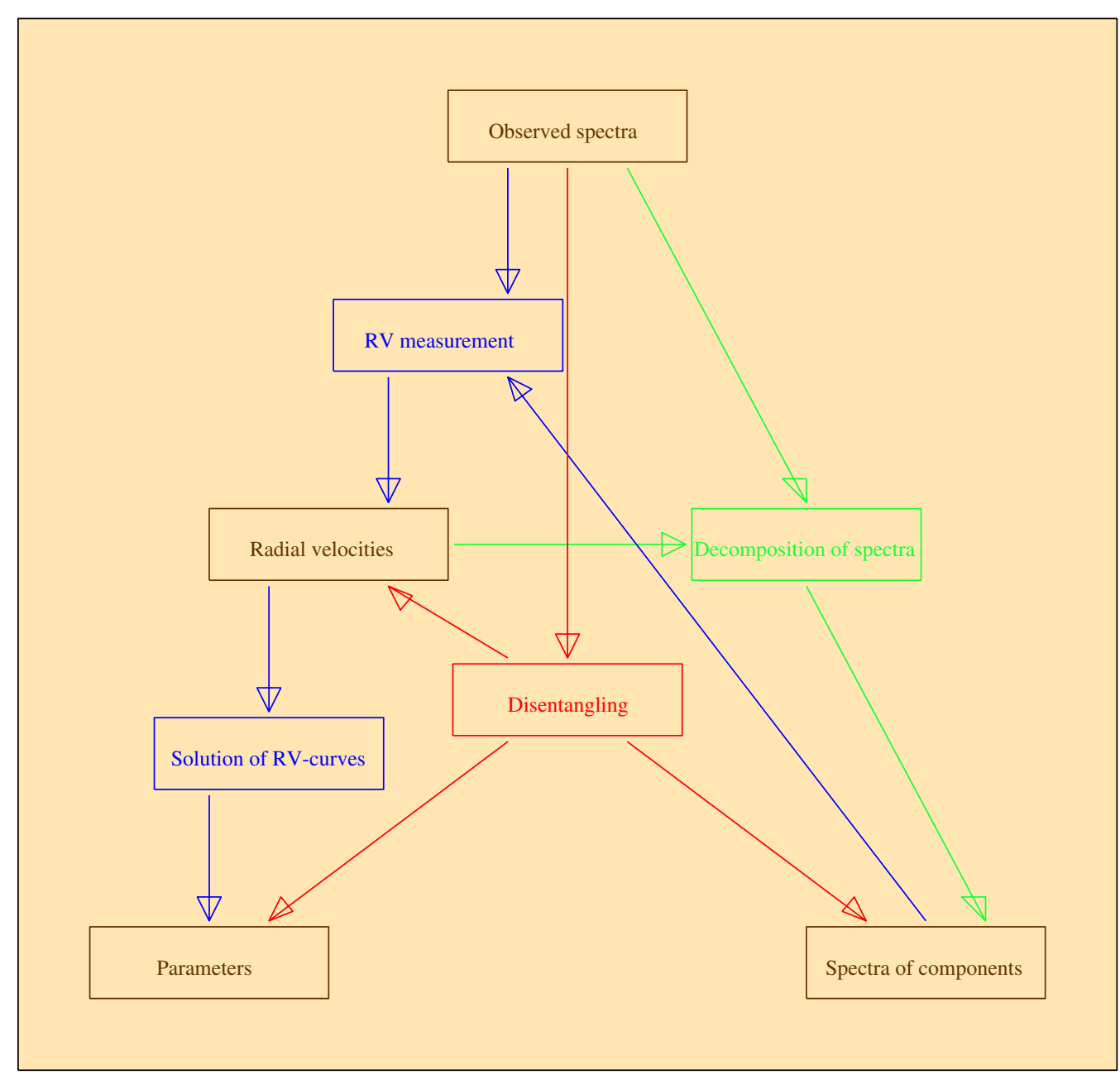

Figure 1. A scheme of data-processing in classical spectroscopic treatment of binaries by RV-curve solution (blue) and spectra decomposition (green), and in disentangling (red)

1994, Hadrava 1995), which comprise both spectra decomposition and RV measurement (or direct parameter fitting). While the decomposition of observed spectra $I(x, t)$ into spectra $I_{j}(x)$ of $n$ component stars is basically a linear problem of solving a huge set (for all logarithmic wavelengths $x=c \ln \lambda$ ) of equations

$$
\sum_{j=1}^{n} I_{j}(x) * \delta\left(x-v_{j}(t, p)\right)=I(x, t)
$$

the fitting of parameters $p$ (or RVs $v_{j}(t)$ ) is non-linear. The whole procedure of disentangling can thus be performed by an iterative minimization of the residual noise of the observed data with respect to the parameters, with decomposition performed in each step. 


\section{Disentangling of spectra or Spectral disentangling ?}

In disentangling we deal with data representing some observed parts of electromagnetic spectrum radiated by the binary (multiple) stellar system. The spectra decomposition can be performed, e.g., by the method of Singular Value Decomposition in the $x$ - representation (Simon and Sturm 1994) or using the Fourier transform method, which reduces the set of Equarions (2.1) into many $n$-dimensional systems for each Fourier component (Hadrava 1995). Recently a kind of iterative procedure for the decomposition was also demonstrated (Gonzáles \& Levato 2006). Mathematical methods which use a representation of solved functions (e.g., for solution of partial differential equations) in a properly chosen system of some basis functions are called Spectral methods. Fourier disentangling thus uses a "spectral" (in the mathematical meaning) decomposition of "spectra" (in physical meaning). However, for any method of disentangling, the generality of the treatment of the data is the most important, not the particular method used. Even for Fourier disentangling it is thus more suitable to speak about "disentangling of spectra" (as it was originally introduced by Simon \& Sturm) or briefly "spectra disentangling", rather than "spectral disentangling".

\section{Disentangling with line-profile variability}

Standard disentangling does not impose any restriction on the component spectra $I_{j}$ apart from their invariability in time (as a consequence, the systemic velocity can be determined only after identifications of lines in the disentangled spectra). However, various changes of line profiles may occur in real multiple stellar systems. These are either phase-locked variations caused by proximity effects, eclipses and circumstellar matter, or some intrinsic line-profile variations on various time-scales due to oscillations, rotation or secular changes of the component stars. In some systems, we can neglect these effects in the first approximation, and study them from $O-C$ as a higher-order perturbation only. But there is a danger of systematic errors e.g., in the disentangled orbital parameters. Moreover, the solution by standard disentangling may fail completely in extreme cases. It is thus desirable to involve the relevant effects directly into the procedure of disentangling.

This can be done by generalization of Equation (2.1) to the form

$$
\sum_{j, k} I_{j}^{k}(x) * \Delta_{j}^{k}(x, t, p)=I(x, t),
$$

where the spectrum of each component star $j$ can be a superposition of several functions $I_{j}^{k}$ corresponding, e.g., to different limb-darkening modes, each one broadened by an appropriate broadening function $\Delta_{j}^{k}$ (cf. Hadrava 1997, 2004c).

The simplest step in this direction consists in involving multiplicative line-strength factors $s_{j}(t)$ into Equation (2.1), as is done in the KOREL04 code (Hadrava 1997, 2004c). This enables to measure eclipses of (not too fast rotating) stars from their spectra, but also to disentangle telluric lines (cf., Hadrava 2006a).

More tricky possibilities are to disentangle rotation of ellipsoidal components (Hadrava \& Kubát 2003) or to disentangle also the Schlessinger - Rossiter - McLaughlin rotational effect in eclipsing binaries (Hadrava 2006c), or oscillations either radial (Hadrava 2004a, 2004c) or non-radial. Other models of line-profile variability, like spots, etc. could be included using a proper model of the stellar disc like in light-curve models (Hadrava 2005). 


\section{Disentangling with constraints}

The advantage of disentangling in unbiased simultaneous searches for orbital parameters and component spectra may turn into a disadvantage, if reliable information on one or the other is available from some other source of data. Generally, all kinds of data (spectroscopy, photometry, astrometry etc.) should be solved simultaneously, as it is enabled e.g., by FOTEL code (cf., Hadrava 2004b). Previous versions of KOREL provided RVs of individual components at each exposure, which could be subsequently included into the FOTEL solution with other RVs (e.g., from the literature), photometry etc. However, the information available in the additional data is not then utilized in the disentangling itself, which can fall into a false solution, or, at least, its convergence is more difficult. In the case of disentangling with LPVs caused by eclipses, the solution of radii or inclination would be safer to perform simultaneously with a light-curve solution (despite the possibility of different photospheric and chromospheric radii which should be considered). Such constraints of parameters can be, in some cases, taken into account by their being fixed at values found from the additional sources. However, more generally, we need to search for a minimum of $(O-C)^{2}$ bound by some conditions $F_{k}(p)=0$ to a subspace of the parameter space. This can be performed by minimization of the sum

$$
S=\sum_{t} \int\left|I-\sum_{j} I_{j} * \Delta_{j}\right|^{2} d x+\sum \lambda_{k} F_{k}^{2}(p)
$$

with some Lagrange multiplicators $\lambda$. Because, in practice, the constraints obtained from the additional data also have some uncertainty, the additional terms $F_{k}^{2}$ can be the $(O-C)^{2}$ for those data and we thus arrive at the problem of simultaneous disentangling and solution of the other data with $\lambda_{\mathrm{s}}$ determining their relative weights, as is done in FOTEL and other similar codes (cf., Holmgren 2004; Hadrava 2004b, 2006b). An important direction is thus a merging of FOTEL and KOREL codes.

A similar situation may concern the component spectra: for a component weak in one spectral region, its spectrum can be better determined by a model corresponding to spectrum disentangled from another region, where the component is stronger. Also the telluric spectrum is quite well known and its disentangling as an unknown function increases the danger of instability, especially of low Fourier modes. The new version of KOREL thus enables to choose some component spectra to be restricted by templates $J_{j}$ given on input and to disentangle the others (Hadrava 2006b), i.e., to solve the equation

$$
\sum_{j=1}^{m} I_{j}(x) * \Delta_{j}(x, t, p)=I(x, t)-\sum_{j=m+1}^{n} J_{j}(x) * \Delta_{j}(x, t, p) .
$$

\section{Conclusions}

Compared with previous methods, spectra disentangling is a step forward towards a complex, general and unbiased interpretation of observational data. Its efficiency has been proven in numerous applications (cf., Holmgren 2004 for review). However, the range of its applicability is not unlimited and users should be aware of the underlying assumptions of the method as well as of possibilities offered by its particular implementation (cf., Hadrava 2004c for a review).

The principle of the method is not exhausted yet and it deserves a further generalization to enlarge its applicability to objects for which it fails or is unreliable at present and to enable a simultaneous fitting of other types of data. 


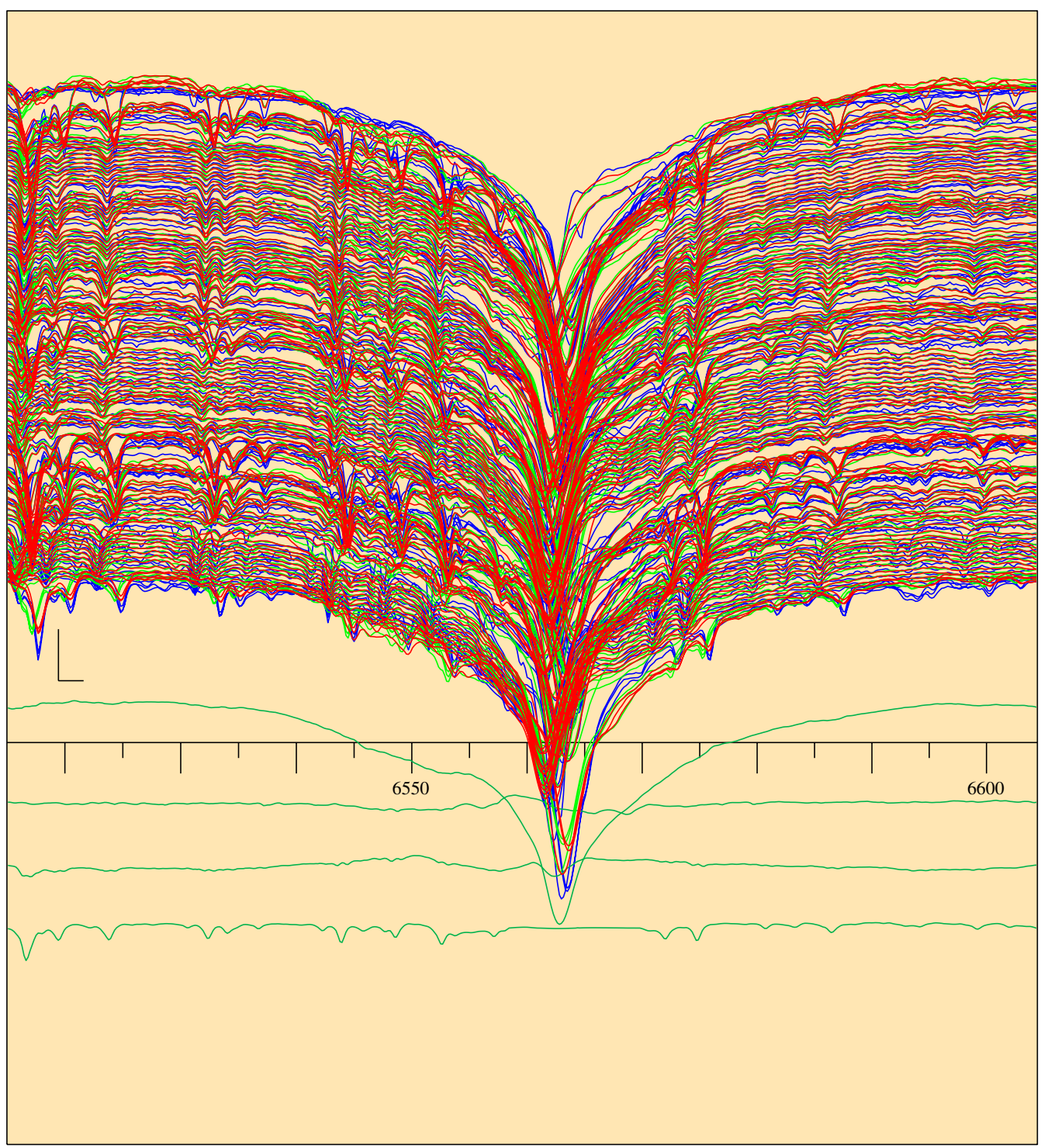

Figure 2. A triple star disentangled with template telluric spectrum artificially straightened in the core of $\mathrm{H} \alpha$ line

\section{Acknowledgements}

The support of this work by grant GA ČR 202/06/0041 and by project AV0Z10030501 is highly appreciated. 


\section{References}

Bagnuolo, W.G. \& Gies, D.R. 1991, ApJ 376, 266

Gonzáles, J.F. \& Levato, H. 2006, A\& $A$ 44, 283

Hadrava, P. 1995, A\&AS 114, 393

Hadrava, P. 1997, A\& AS 122, 581

Hadrava, P. 2004a, in: Spectroscopically and Spatially Resolving the Components of the Close Binary Stars, R.W. Hilditch, H. Hensberge \& K. Pavlovski, eds., ASP Conf. Ser. 318, p. 86

Hadrava, P. 2004b, Publ. Astron. Inst. ASCR 92, 1

Hadrava, P. 2004c, Publ. Astron. Inst. ASCR 92, 15

Hadrava, P. 2005, ApESSS 296, 239

Hadrava, P. 2006a, A\&A 448, 1149

Hadrava, P. 2006b, ApESSS, in press

Hadrava, P. 2006c, ASP Conf. Ser., submitted

Hadrava, P. \& Kubát, J. 2003, in: Stellar Atmosphere Modeling, I. Hubeny, D. Mihalas \& K. Werner, eds., ASP Conf. Ser. 288, p. 149

Hill, G. 1993, in: New Frontiers in Binary Stars Research, K.-C. Leung \& I.-S. Nhu, eds., ASP Conf. Ser. 38, p. 127

Holmgren, D. 2004, in: Spectroscopically and Spatially Resolving the Components of the Close Binary Stars, R. W. Hilditch, H. Hensberge \& K. Pavlovski eds., ASP Conf. Ser. 318, p. 95

Kallrath, J. \& Milone, E.F. 1999, Eclipsing Binary Stars: Modeling and Analysis, SpringerVerlag, New York, Berlin

Rucinski, S. 1992, AJ 104, 1968

Simon, K.P. \& Sturm, E. 1994, A\&A 281, 286

Wilson, R.E. 1979, ApJ 234, 1054

Zucker, S. \& Mazeh, T. 1994, ApJ 420, 806 